Pacific Journal of Mathematics

DIAGONABILITY OF IDEMPOTENT MATRICES 


\title{
DIAGONABILITY OF IDEMPOTENT MATRICES
}

\section{Arthur STEGER}

\begin{abstract}
A ring $\mathscr{R}$ (commutative with identity) with the property that every idempotent matrix over $\mathscr{R}$ is diagonable (i.e., similar to a diagonal matrix) will be called an $I D$-ring. We show that, in an $I D$-ring $\mathscr{R}$, if the elements $a_{1}, a_{2}, \cdots, a_{n} \in \mathscr{R}$ generate the unit ideal then the vector $\left[a_{1}, a_{2}, \cdots, a_{n}\right]$ can be completed to an invertible matrix over $\mathscr{R}$. We establish a canonical form (unique with respect to similarity) for the idempotent matrices over an $I D$-ring. We prove that if $\mathscr{N}^{\prime}$ is the ideal of nilpotents in $\mathscr{R}$ then $\mathscr{R}$ is an $I D$-ring if and only if $\mathscr{R} / \mathscr{N}$ is an $I D$-ring. The following are then shown to be $I D$-rings: elementary divisor rings, a restricted class of Hermite rings, $\pi$-regular rings, quasi-semi-local rings, polynomial rings in one variable over a principal ideal ring (zero divisors permitted), and polynomial rings in two variables over a $\pi$-regular ring with finitely many idempotents.
\end{abstract}

In this paper, $\mathscr{R}$ will denote a commutative ring with identity, and $\mathscr{R}_{n}$ will denote the set of $n \times n$ matrices over $\mathscr{R}$. If $A, B \in \mathscr{R}_{n}$, then $A \cong B$ will mean that $A$ is similar to $B$. We remark that if $\mathscr{R}$ is an $I D$-ring then every finitely generated projective $R$-module is the finite direct sum of cyclic modules, and that $\mathscr{R}$ is a directly indecomposable $I D$-ring if and only if every finitely generated projective $\mathscr{R}$-module is free. Most of the literature on this subject has been concerned with showing that a given ring $\mathscr{R}$ has the property that every finitely generated projective $\mathscr{R}$-module is free. This necessarily imposes the condition that $\mathscr{R}$ be indecomposable. In this paper, no such restriction is made.

\section{Properties of ID-rings.}

DeFINITION 1. $\mathscr{R}$ is said to be an $I D$-ring provided that for every $A=A^{2} \in \mathscr{R}_{n}, n=1,2, \cdots$, there exists an invertible matrix $P \in \mathscr{R}_{n}$ such that $P A P^{-1}$ is a diagonal matrix.

DeFinition 2. The row vector $\left[a_{1}, a_{2}, \cdots, a_{n}\right]$ with components in $\mathscr{R}$ is said to be a basal provided that it can be completed to an invertible matrix over $\mathscr{R}$.

DEFINITION 3. The row vector $X$ is said to be a characteristic vector of $A \in \mathscr{R}_{n}$ corresponding to $r \in \mathscr{R}$ provided (1) $X$ is a basal vector and (2) $X A=r X$. 
The following lemma, due to A. L. Foster, is an important tool in our development.

Foster's LemMa. $\mathscr{R}$ is an ID-ring if and only if every idempotent matrix over $\mathscr{R}$ has a characteristic vector.

From this lemma, which appears essentially as Theorem 10 in [2], one can quickly deduce that quasi-local rings and principal ideal domains are ID-rings. Then, known structure theorems suffice to show that principal ideal rings (see [7], p. 66), rings with descending chain condition, and Boolean rings are $I D$. These results will be extended in the next section.

THEOREM 1. Let $A=A^{2} \in \mathscr{R}_{n}$. If there exist invertible matrices $P, Q \in \mathscr{R}_{n}$ such that $P A Q$ is a diagonal matrix then $A$ is diagonable.

Proof. Let $P A Q=B=\operatorname{diag}\left(b_{1}, b_{2}, \cdots, b_{n}\right)$ and let $U=Q^{-1} P^{-1}=$ $\left(u_{i j}\right)$. Then $(B U)^{2}=B U$ and $B U B=B$. Hence $b_{i}=b_{i}^{2} u_{i i}, b_{i} u_{i i}$ is idempotent, and by Lemma 2.1 of [9] $b_{i} \sim b_{i} u_{i i}$ for each $i$. Thus, we may assume that $Q$ has been adjusted so that $b_{i}^{2}=b_{i}, i=1,2, \cdots, n$. The equation $B U B=B$ now yields

(1) $b_{i} u_{i i}=b_{i}, i=1,2, \cdots, n$, and

(2) $b_{i} b_{j} u_{i j}=0, i \neq j, i, j=1,2, \cdots, n$.

From (1),

$$
B U=\left[\begin{array}{llll}
b_{1} & b_{1} u_{12} & \cdots & b_{1} u_{1 n} \\
b_{2} u_{21} & b_{2} & \cdots & b_{2} u_{2 n} \\
\vdots & \vdots & & \vdots \\
b_{n} u_{n 1} & b_{n} u_{n 2} & \cdots & b_{n}
\end{array}\right]
$$

If $X_{k}=\left[b_{k} u_{k 1}, b_{k} u_{k 2}, \cdots, b_{k} u_{k k-1}, 1, b_{k} u_{k k+1}, \cdots, b_{k} u_{k n}\right]$ then $X_{k} B U=$ $b_{k} X_{k}, k=1,2, \cdots, n$. Now let

$$
C=\left[\begin{array}{c}
X_{1} \\
X_{2} \\
\vdots \\
X_{n}
\end{array}\right]
$$

From (2), it follows that $|C|=1$. Hence $(C P) A(C P)^{-1}=C B U C^{-1}=$ $\operatorname{diag}\left(b_{1}, b_{2}, \cdots, b_{n}\right)$.

THEOREM 2. Let $\mathscr{R}$ be an ID-ring. If $a_{1}, a_{2}, \cdots, a_{n} \in \mathscr{R}$ generate the unit ideal in $\mathscr{R}$ then the vector $\left[a_{1}, a_{2}, \cdots, a_{n}\right]$ is basal. 
Proof. Let $\sum_{i=1}^{n} x_{i} a_{i}=1$ and let $B=\left(x_{i} a_{j}\right) \in \mathscr{R}_{n}$. Then $B^{2}=B$ and $\operatorname{tr} B=1$. Since $\mathscr{R}$ is $I D, B \cong C=\operatorname{diag}\left(c_{1}, c_{2}, \cdots, c_{n}\right)$. If $X=$ $\left[c_{1}, c_{2}, \cdots, c_{n}\right]$ then $X C=X$ and, since $\sum_{i=1}^{n} c_{i}=1$,

$$
\left|\begin{array}{lllll}
c_{1} & c_{2} & c_{3} & \cdots & c_{n} \\
-1 & 1 & 0 & \cdots & 0 \\
-1 & 0 & 1 & \cdots & 0 \\
\vdots & \vdots & \vdots & & \vdots \\
-1 & 0 & 0 & \cdots & 1
\end{array}\right|=1
$$

Hence, $B$ has a characteristic vector $Y=\left[y_{1}, y_{2}, \cdots, y_{n}\right]$ corresponding to 1. From $Y B=Y$, we have $\sum_{i=1}^{n} y_{i} x_{i} a_{j}=y_{j}, j=1,2, \cdots, n$. Thus $\left(\sum_{i=1}^{n} y_{i} x_{i}\right)\left[a_{1}, a_{2}, \cdots, a_{n}\right]=\left[y_{1}, y_{2}, \cdots, y_{n}\right]$. Since $Y$ is basal, so also is $\left[a_{1}, a_{2}, \cdots, a_{n}\right]$.

Theorem 3. If $\mathscr{R}$ is an ID-ring then every invertible ideal in $\mathscr{R}$ is principal.

Proof. Let $\mathscr{C}$ be an invertible ideal in $\mathscr{R}$. Then there exist elements $a_{1}, a_{2}, \cdots, a_{n} \in \mathscr{K}$ and elements $x_{1}, x_{2}, \cdots, x_{n}$ in the full ring of quotients of $\mathscr{R}$ such that $x_{i} \mathscr{K} \subseteq \mathscr{R}, i=1,2, \cdots, n$, and $\sum_{i=1}^{n} x_{i} a_{i}=1$. It follows that $\mathscr{K}=\left(a_{1}, a_{2}, \cdots, a_{n}\right)$. Let $B=\left(x_{i} a_{\jmath}\right) \in \mathscr{R}_{n}$. Then, as in Theorem 2 , there exists a basal vector $Y=\left[y_{1}, y_{2}, \cdots, y_{n}\right]$ such that $y_{j}=\sum_{i=1}^{n} y_{i} x_{i} a_{\jmath}, j=1,2, \cdots, n$. Now let $x_{i}=c_{\imath} / d, c_{i}, d \in \mathscr{R}$ and $d$ not a zero divisor. If $p=\sum_{i=1}^{n} y_{i} c_{i}$ then $\left[p a_{1}, p a_{2}, \cdots, p a_{n}\right]=$ $\left[d y_{1}, d y_{2}, \cdots, d y_{n}\right]$. Since $Y$ is basal, $p \mathscr{K}=(d)$. Hence there is an $a \in \mathscr{K}$ such that $p a=d$. Thus, $p$ is not a zero divisor. If $b \in \mathscr{K}$, then for some $r \in \mathscr{R}, p b=r d=p r a$. Hence, $b=r a$ and $\mathscr{K}=(a)$.

Recall that if $\mathscr{S}$ is the set of idempotents of $\mathscr{R}$ then $\langle\mathscr{S}, \cap$, $\cup, *>$ where $a \cap b=a b, a \cap b=a+b-a b$, and $a^{*}=1-a$, is a Boolean algebra (see [1]). It follows that if $a_{1}, a_{2}, \cdots, a_{n} \in \mathscr{S}$ and $a=\bigcup_{i=1}^{n} \alpha_{1}$ then $a_{1}, a_{2}, \cdots, a_{n}$ generate the principal ideal $(a)$ in $\mathscr{R}$.

Theorem 4. (Canonical Form) Let $\mathscr{R}$ be an ID-ring and let $A=A^{2} \in \mathscr{R}_{n}$. Then $A \cong \operatorname{diag}\left(a_{1}, a_{2}, \cdots, a_{n}\right) \quad$ where $a_{i} \mid a_{i+1}, i=$ $1,2, \cdots, n-1$. Moresver, if $A \cong \operatorname{diag}\left(b_{1}, b_{2}, \cdots, b_{n}\right)$ with $b_{i} \mid b_{i+1}$, $i=1,2, \cdots, n-1$, then $a_{i}=b_{i}, i=1,2, \cdots, n$.

Proof. Since $\mathscr{R}$ is $I D$, let $A \cong C=\operatorname{diag}\left(c_{1}, c_{2}, \cdots, c_{n}\right)$ and let $a_{1}=\bigcup_{i=1}^{n} e_{i}$. Then there exist idempotents $x_{1}, x_{2}, \cdots, x_{n}$ such that $x_{\imath} a_{1}=c_{i}$ for each $i$ and $\bigcup_{i=1}^{n} x_{i}=1$. Thus, $\left(x_{1}, x_{2}, \cdots, x_{n}\right)=1$ and, by Theorem 2, $X=\left[x_{1}, x_{2}, \cdots, x_{n}\right]$ is basal. Since $x_{i}$ is idempotent, $i=1,2, \cdots, n, X C=a_{1} X$ and, as in the proof of Foster's Lemma, 
$A \cong \operatorname{diag}\left(a_{1}, d_{2}, \cdots, d_{n}\right)$. By induction, $A \cong \operatorname{diag}\left(a_{1}, a_{2}, \cdots, a_{n}\right)$ where $a_{i} \mid a_{i+1}, i=2,3, \cdots, n-1$. Since $a_{1}$ divides each entry of $C, a_{1} \mid a_{2}$. If also, $A \cong \operatorname{diag}\left(b_{1}, b_{2}, \cdots, b_{n}\right)$ with $b_{i} \mid b_{i+1}, i=1,2, \cdots, n-1$, then it is a consequence of Theorem 9.3 of [6] that $b_{i}=a_{i}$ for each $i$. This can also be seen directly as follows: since $a_{r}$ divides each $r$-rowed minor of diag $\left(a_{1}, a_{2}, \cdots, a_{n}\right), a_{r}$ divides $b_{r}=b_{i} b_{2} \cdots b_{r}$. Similarly, $b_{r}$ divides $a_{r}$ and, since both $a_{r}$ and $b_{r}$ are idempotent, $a_{r}=b_{r}, r=$ $1,2, \cdots, n$.

Corollary. If $\mathscr{R}$ is ID and $A=A^{2} \in \mathscr{R}_{n}$ then $A$ has a characteristic vector corresponding to $|A|$.

Proof. We need merely observe that if $A \cong \operatorname{diag}\left(a_{1}, a_{2}, \cdots, a_{n}\right)$ with $a_{i} \mid a_{i+1}, i=1,2, \cdots, n-1$, then $a_{n}=|A|$.

THeORem 5. Let $\mathscr{J}$ be the Jacobson radical of $\mathscr{R}$, let $\mathscr{N}$ be the ideal of nilpotents in $\mathscr{R}$, and let $\mathscr{K}$ be an arbitrary ideal in $\mathscr{R}$. If $\mathscr{K} \cong \mathscr{J}$ and $\mathscr{R} / \mathscr{K}$ is an ID-ring then $\mathscr{R}$ is an ID-ring. If $\mathscr{K} \subseteq \mathscr{N}$ then $\mathscr{R}$ is an ID-ring if and only if $\mathscr{R} / \mathscr{K}$ is an ID-ring.

Proof. Let $\mathscr{C} \cong \mathscr{J}$ and assume that $\mathscr{R} / \mathscr{K}$ is $I D$. Let $A=$ $A^{2}=\left(A_{i j}\right) \in \mathscr{R}_{n}$ and $A^{*}=\left(a_{i j}+\mathscr{K}\right)$. Then $\left(A^{*}\right)^{2}=A^{*}$ and if $d=$ $|A|$ then $d+\mathscr{C}=\left|A^{*}\right|$. By the corollary to Theorem 4, we may let $X^{*}=\left[x_{1}+\mathscr{K}, x_{2}+\mathscr{K}, \cdots, x_{n}+\mathscr{K}\right]$ be a characteristic vector of $A^{*}$ corresponding to $d+\mathscr{K}$. Then, if $X=\left[x_{1}, x_{2}, \cdots, x_{n}\right], X A=$ $d X+Y$ where the components of $Y$ are in $\mathscr{K}$. Since $A^{2}=A$ and $d^{2}=d, X A=d X A+Y A, \quad Y A=(1-d) X A=(1-d) Y, \quad$ and $(X+(2 d-1) Y) A=d X+d Y=d(X+(2 d-1) Y)$. Since $\mathscr{K} \cong \mathscr{J}$, $u+\mathscr{K}$ is a unit of $\mathscr{R} / \mathscr{K}$ if and only if $u$ is a unit of $\mathscr{R}$. It follows, therefore, that since $X^{*}$ is basal so also is $X+(2 d-1) Y$. By Foster's Lemma, $\mathscr{R}$ is $I D$. Now let $\mathscr{K} \cong \mathscr{N}$. Since $\mathscr{N} \subseteq \mathscr{J}$, we need only prove that if $\mathscr{R}$ is $I D$ then $\mathscr{R} / \mathscr{K}$ is $I D$. Hence, assume that $\mathscr{R}$ is $I D$ and $A^{*}=\left(A^{*}\right)^{2}=\left(a_{i j}+\mathscr{K}\right) \in(\mathscr{R} / \mathscr{K})_{n}$. It will suffice to show that there exists an idempotent matrix $F=$ $\left(f_{i j}\right) \in \mathscr{R}_{n}$ such that $f_{i j}+\mathscr{K}=a_{i j}+\mathscr{K}, i, j=1,2, \cdots, n$. If $A=$ $\left(a_{i j}\right)$ then $A^{2}=A+B$ where the components of $B$ are in $\mathscr{K}$. Thus $B$ is nilpotent. Let $k$ be the least natural number such that $B^{k}=$ $Z=$ zero matrix. If $k=1$, there is nothing left to prove. Hence, assume that $k>1$ and let $C=A+(I-2 A) B$. Then the components of $C-A$ are in $K$ and, since $A B=B A$,

$$
C^{2}=A^{2}+2 A(I-2 A) B+(I-2 A)^{2} B^{2} .
$$

Therefore, $C^{2}-C=B+(I-2 A)^{2}\left(B^{2}-B\right)$. Since $(I-2 A)^{2}=I+4 B$, 
$C^{2}=C+B^{2}(4 B-3 I)$. If we let $D=B^{2}(4 B-3 I)$, we have $C^{2}=$ $C+D$ where the components of $D$ are in $\mathscr{C}$ and, for some natural number $l<k, D^{l}=Z$. Repeating this process, we arrive in a finite number of steps at the required matrix $F$.

Corrollary. Let $\mathscr{N}$ be the ideal of nilpotents in $\mathscr{R}$ and let $x_{1}, x_{2}, \cdots, x_{k}$ be indeterminates. Then $\mathscr{R}\left[x_{1}, x_{2}, \cdots, x_{k}\right]$ is ID if and only if $(\mathscr{R} / \mathscr{N})\left[x_{1}, x_{2}, \cdots, x_{k}\right]$ is $I D$.

Proof. The corollary follows by observing that $\mathscr{N}\left[x_{1}, x_{2}, \cdots, x_{k}\right]$ is the ideal of nilpotents in $\mathscr{R}\left[x_{1}, x_{2}, \cdots, x_{k}\right]$ and that

$$
\mathscr{R}\left[x_{1}, x_{2}, \cdots, x_{k}\right] / \mathscr{N}\left[x_{1}, x_{2}, \cdots, x_{k}\right] \approx(\mathscr{R} / \mathscr{N})\left[x_{1}, x_{2}, \cdots, x_{k}\right] .
$$

3. Classes of ID-rings. As an immediate consequence of Theorem 1, we have:

THEOREM 6. An elementary divisor ring is an ID-ring.

THEOREM 7. Let $\mathscr{R}$ be a Hermite ring with Jacobson radical $\mathcal{J}$. If $\mathscr{R}$ has the property that $a b=0$ implies either $(a)=\left(a^{2}\right)$ or $a \in \mathscr{J}$ or $b \in \mathcal{J}$ then $\mathscr{R}$ is an ID-ring.

Proof. Let $A=A^{2}=\left(a_{i j}\right) \in \mathscr{R}_{n}$ and let $Q$ be an invertible matrix such that $Q A=B=\left(b_{i j}\right)$ is triangular; i.e., $b_{i j}=0$ if $i<j$. Let $Q^{-1}=\left(p_{i j}\right)$. Then $X=\left[b_{11} p_{11}, b_{11} p_{12}, \cdots, b_{11} p_{1 n}\right]$ is the first row of $Q A Q^{-1}$. If $\left(b_{11}\right)=\left(b_{11}^{2}\right)$ then there is an idempotent $e$ such that $b_{11} \sim e$. By Theorem 3.9 of [6], there are vectors $X_{2}, X_{3}, \cdots, X_{n}$ such that

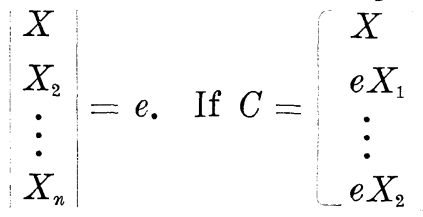

then $|C+(1-e) I|=1$. Thus, the vector

$Y=\left[b_{11} p_{11}+1-e, b_{11} p_{12}, \cdots, b_{11} p_{1 n}\right]$ is basal and $Y\left(Q A Q^{-1}\right)=X=$ $e X=e Y$; i.e, $Y$ is a characteristic vector of $Q A Q^{-1}$ corresponding to $e$. If $b_{11} \in \mathscr{J}$ then $1-b_{11} p_{11}$ is a unit of $\mathscr{R}$ and

$$
\left[1-b_{11} p_{11},-b_{11} p_{12}, \cdots,-b_{11} p_{1 n}\right]
$$

is a characteristic vector of $Q A Q^{-1}$ corresponding to 0 . Suppose now that neither of these assumptions on $b_{11}$ is true. From the equation, $B A=Q A^{2}=Q A=B$, we obtain $b_{11}\left(1-a_{11}\right)=0$. By the hypothesis on $\mathscr{R}, 1-a_{11} \in \mathscr{J}, a_{11}$ is a unit of $\mathscr{R}$, and $\left[a_{11}, a_{12}, \cdots, a_{1 n}\right]$ is a characteristic vector of $A$ corresponding to 1 . In any event, $A$ has a characteristic vector and Foster's Lemma completes the proof. 
THEOREM 8. A $\pi$-regular ring is an ID-ring.

Proof. Let $\mathscr{R}$ be $\pi$-regular with Jacobson radical $\mathscr{J}$. Then $\mathscr{R} / \mathscr{J}$ is regular and, therefore an elementary divisor ring (see [3], p. 365). The conclusion follows from Theorems 5 and 6 .

Theorem 9. A quasi-semi-local ring is an ID-ring.

Proof. Let $\mathscr{R}$ be quasi-semi-local with Jacobson radical $\mathscr{J}$. Since, by definition, $\mathscr{R}$ has only a finite number of maximal ideals, $\mathscr{R} / \mathscr{J}$ is a finite direct sum of fields. Theorem 5 completes the proof.

Theorem 10. Let $\mathscr{R}$ be an ID-ring and let $\mathscr{S}$ be a subring of $R[[x]]$ which contains $\mathscr{R}$. If $\mathscr{S}$ has the property that $u \in \mathscr{S}$ and $u$ is a unit of $\mathscr{R}[[x]]$ imply that $u$ is a unit of $\mathscr{S}$ then $\mathscr{S}$ is an ID-ring.

Proof. Let $A=A^{2} \in \mathscr{S}_{n}$ and let $A^{\prime}$ be the matrix in $\mathscr{R}_{n}$ obtained from $A$ by suppressing all positive powers of $x$. If $A^{\prime}=Z=$ zero matrix and $A \neq Z$, let $k$ be the highest power of $x$ which divides (in $R[[x]])$ each entry in $A$. Then we may write $A=x^{k} B$; and some entry in $B$ is not divisible by $x$. Since $A$ is idempotent $x^{k} B=x^{2 k} B^{2}$. Thus, $B=x^{k} B^{2}$ and, since $k>0$, we have arrived at a contradiction. Again, let $A=A^{2} \in \mathscr{S}_{n}$. Then $\left(A^{\prime}\right)^{2}=A^{\prime}$ and, since $\mathscr{R}$ is $I D$, it follows from Theorem 4 that the entries of $A^{\prime}$ generate in $\mathscr{R}$ a principal ideal $(e)$ where $e$ is idempotent. Then $(1-e) A$ is idempotent and $((1-e) A)^{\prime}=Z$. Thus, $(1-e) A=Z$. Let $P$ be an invertible matrix in $\mathscr{K}_{n}$ such that $P A^{\prime} P^{-1}=\operatorname{diag}\left(a_{1}, a_{2}, \cdots, a_{n}\right)$ where $a_{i} \mid a_{i+1}$, $i=1,2, \cdots, n-1$. Therefore, $a_{1}=e$ and $P A P^{-1}=B=\left(b_{i j}\right)$ with $b_{11}=e+r_{1} x+r_{2} x^{2}+\cdots$. Then, if $Y=\left[1-e+b_{11}, b_{12}, \cdots, b_{1 n}\right]$, $(1-e) B=Z$ implies $Y B=e Y$. Since $1-e+b_{11}$ is a unit in $R[[x]]$, by the hypothesis on $\mathscr{S}, Y$ is a characteristic vector corresponding to $e$. The theorem follows from Foster's lemma.

Theorem 10 shows for example that the domain of complex valued functions of a complex variable which are analytic at some point $z_{0}$ in the complex plane is an $I D$-ring, or that the domain of real valued functions of a real variable analytic at some real number $r_{0}$ is $I D$. It is also true that the domain of entire functions is $I D$. This has, however, nothing to with Theorem 10; but it is rather a consequence of Theorem 7 in conjunction with a theorem proved in [4] to the effect that in the domain of entire functions every finitely generated ideal is principal. 
The problem of determining, given a ring $\mathscr{R}$, whether or not $\mathscr{R}[x]$ is $I D$ is a difficult one. An important result in this area is due to Seshadri who proved in [8] that if $\mathscr{R}$ is a principal ideal domain then $\mathscr{R}[x]$ is $I D$. In particular, $\mathscr{K}[x, y]$, where $\mathscr{K}$ is a field, is $\mathscr{I} D$. The character of $\mathscr{K}[x, y, z]$ is open. Horrocks showed ([5]), p. 718) that if $\mathscr{R}$ is a regular local ring of dimension 2 with a field of coefficients then $\mathscr{R}[x]$ is $I D$. Chase, on the other hand, has constructed an example (unpublished) of a complete local domain $\mathscr{R}$ such that $\mathscr{R}[x]$ is not $I D$. The ring in Chase's example has dimension 1 , is not a regular local ring, and in fact is not integrally closed.

THEOREM 11. Let $\mathscr{R}$ be a ring with $\mathscr{N}$ its ideal of nilpotents. (1) If $\mathscr{R} / \mathscr{N}$ is a principal ideal ring then $\mathscr{R}[x]$ is $I D$; (2) if $\mathscr{R} / \mathscr{N}$ is a Boolean ring then $\mathscr{R}[x, y]$ is ID; and (3) if $\mathscr{R}$ is a $\pi$ regular ring with finitely many idempotents then $\mathscr{R}[x, y]$ is $I D$.

Proof. The assertions of this theorem are a consequence of applying the Corollary to Theorem 5 to Seshadri's result. First, assume that $\mathscr{R} / \mathscr{N}$ is a principal ideal ring. It is a consequence of the result on page 66 of [7] that $\mathscr{R} / \mathscr{N}$ is a finite direct sum of principal ideal domains. Thus (1) has been established. Now assume that $\mathscr{R} / \mathscr{N}$ is a Boolean ring and let $A=A^{2} \in((\mathscr{R} / \mathscr{N})[x, y])_{n}$. Then the set of coefficients of the entries in $A$ together with 1 generate a finite Boolean subring $\mathscr{S}$ of $\mathscr{R} / \mathscr{N}$ whose unit element is the unit element of $\mathscr{R} / \mathscr{N}$. Since $\mathscr{S}$ is the finite direct sum of fields, $A$ is diagonable and (2) has been proved. Finally, assume that $\mathscr{R}$ is a $\pi$-regular ring with finitely many idempotents. Then $\mathscr{R} / \mathscr{N}$ is the finite direct sum of fields. This completes the proof of (3).

\section{REFERENCES}

1. A. L. Foster, The idempotents elements of a commutative ring form a Boolean algebra, Duke Math. J. 12 (1945), 143-152.

2. Maximal idempotent sets in a ring with unit, Duke Math J. 13 (1946), 247-58.

3. L. Gilman and M. Henriksen, Some remarks about elementary divisor rings, Trans. Amer. Math. Soc. 82 (1956), 362-365.

4. O. Helmer, Divisibility properties of integral functions, Duke Math. J. 6 (1940), 345-356.

5. G. Horrocks, Projective modules over an extension of a local ring, London Math. Soc. (3) 14 (1964), 714-718.

6. I. Kaplansky, Elementary divisors and modules, Trans. Amer. Math. Soc. 66 (1949), 464-491.

7. G. Pollak, Über die Structur Kommutative Hauptideatringe, Acta. Sci. Math. 22 (1961), 62-74. 
8. C. S. Seshadri, Triviality of vector bundles over affine space $K^{2}$, Proc. Nat. Acad. of Sci. USA 44 (1958), 456-458.

9. A. Steger, Elementary factorization in $\pi$-regular rings (to appear in the Canad. J. Math.)

Received July 8, 1965.

University of NeW MeXico 


\section{PACIFIC JOURNAL OF MATHEMATICS}

\section{EDITORS}

H. SAMELSON

Stanford University

Stanford, California

J. P. JANS

University of Washington

Seattle, Washington 98105

\section{J. DuGUNDJI}

University of Southern California

Los Angeles, California 90007

RICHARD ARENS

University of California

Los Angeles, California 90024

\section{ASSOCIATE EDITORS}

E. F. BECKENBACH
B. H. NEUMANN

F. WOLF

K. YoSIDA

\section{SUPPORTING INSTITUTIONS}

\author{
UNIVERSITY OF BRITISH COLUMBIA \\ CALIFORNIA INSTITUTE OF TECHNOLOGY \\ UNIVERSITY OF CALIFORNIA \\ MONTANA STATE UNIVERSITY \\ UNIVERSITY OF NEVADA \\ NEW MEXICO STATE UNIVERSITY \\ OREGON STATE UNIVERSITY \\ UNIVERSITY OF OREGON \\ OSAKA UNIVERSITY \\ UNIVERSITY OF SOUTHERN CALIFORNIA
}

\author{
STANFORD UNIVERSITY \\ UNIVERSITY OF TOKYO \\ UNIVERSITY OF UTAH \\ WASHINGTON STATE UNIVERSITY \\ UNIVERSITY OF WASHINGTON \\ AMERICAN MATHEMATICAL SOCIETY \\ CHEVRON RESEARCH CORPORATION \\ TRW SYSTEMS \\ NAVAL ORDNANCE TEST STATION
}

Mathematical papers intended for publication in the Pacific Journal of Mathematics should be typewritten (double spaced). The first paragraph or two must be capable of being used separately as a synopsis of the entire paper. It should not contain references to the bibliography. Manuscripts may be sent to any one of the four editors. All other communications to the editors should be addressed to the managing editor, Richard Arens at the University of California, Los Angeles, California 90024 .

50 reprints per author of each article are furnished free of charge; additional copies may be obtained at cost in multiples of 50 .

The Pacific Journal of Mathematics is published monthly. Effective with Volume 16 the price per volume (3 numbers) is $\$ 8.00$; single issues, $\$ 3.00$. Special price for current issues to individual faculty members of supporting institutions and to individual members of the American Mathematical Society: $\$ 4.00$ per volume; single issues $\$ 1.50$. Back numbers are available.

Subscriptions, orders for back numbers, and changes of address should be sent to Pacific Journal of Mathematics, 103 Highland Boulevard, Berkeley 8, California.

Printed at Kokusai Bunken Insatsusha (International Academic Printing Co., Ltd.), No. 6, 2-chome, Fujimi-cho, Chiyoda-ku, Tokyo, Japan.

\section{PUBLISHED BY PACIFIC JOURNAL OF MATHEMATICS, A NON-PROFIT CORPORATION}

The Supporting Institutions listed above contribute to the cost of publication of this Journal, but they are not owners or publishers and have no responsibility for its content or policies. 


\section{Pacific Journal of Mathematics

Vol. 19, No. $3 \quad$ July, 1966

S. J. Bernau, The spectral theorem for unbounded normal operators .......

Lu-san Chen, Asymptotic behavior of solutions of parabolic equations of

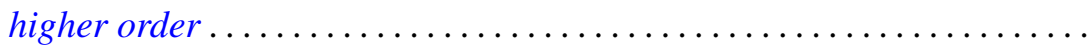

Lawrence William Conlon, An application of the Bott suspension map to the

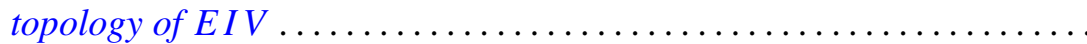

Neal Eugene Foland and John M. Marr, Sets with zero-dimensional kernels .........................................

Stanley Phillip Franklin and R. H. Sorgenfrey, Closed and image-closed

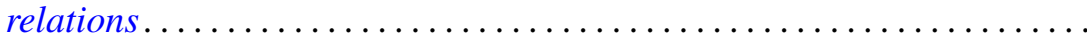

William Jesse Gray, A note on topological transformation groups with a

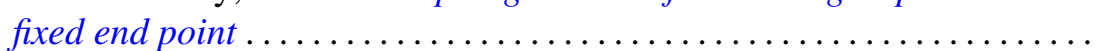

Myron Goldstein, $K$ - and L-kernels on an arbitrary Riemann surface ...... 449

George Joseph Kertz and Francis Regan, The exponential analogue of a generalized Weierstrass series .............................

Walter Leighton, On Liapunov functions with a single critical point ........ 467

Bernard Werner Levinger and Richard Steven Varga, On a problem of $O$.

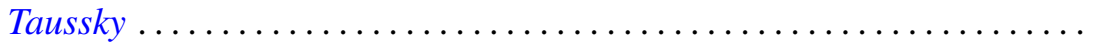

Lowell Duane Loveland, Tame subsets of spheres in $E^{3} \ldots \ldots \ldots \ldots \ldots . .489$

Erik Andrew Schreiner, Modular pairs in orthomodular lattices ......... 519

K. N. Srivastava, On dual series relations involving Laguerre polynomials ...............................

Arthur Steger, Diagonability of idempotent matrices.....

Walter Strauss, On continuity of functions with values in various Banach spaces...

Robert Vermes, On the zeros of a linear combination of polynomials ...

Elliot Carl Weinberg, On the scarcity of lattice-ordered matrix rings ....

Harold Widom, Toeplitz operators on $H_{p} \ldots \ldots \ldots \ldots \ldots$

Neal Zierler, On the lattice of closed subspaces of Hilbert space...

Irving Leonard Glicksberg, Correction to: "Maximal algebras and a theorem of Rado"

John Spurgeon Bradley, Correction to: "Adjoint quasi-differential operators of Euler type"

William Branham Jones, Erratum: "Duality and types of completeness in locally covex spaces".

Stanley P. Gudder, Erratum: "Uniqueness and existence properties of bounded observables" 